\title{
Transplanted dopaminergic neurons: More or less?
}

To the efitar-Much debate has been generated from the results recently published by Freed et al. $^{1}$ describing the outcome of the first double-blind placebo-controlled clinical trial on the efficacy of embryonic neural tissue transplantation in Parkinson disease. Particular interest has been focused on the severe dyskinetic side effects observed in several patients in the study, from which the authors drew the conclusion, highlighted by your editorial ${ }^{2}$, that "transplanting less fetal tissue in the future" would be the simplest way to increase the clinical efficacy and safety of neural transplantation in Parkinson disease patients. We question both this interpretation and the remedy offered.

This study shows modest symptomatic improvements in younger patients at one year after transplantation. The necessity to improve the safety of their transplantation method was based on the observation that 5 of 33 transplanted patients developed dyskinesias and facial dystonias, even in the absence of medication. These were interpreted as resulting from "a relative excess of dopamine" derived from "too many" grafted neurons, in direct analogy to peak-dose dyskinesias seen with L-dopa medication. However, a more careful analysis of the post mortem data obtained from two of their patients reveals a number of facts that may point in another direction. Each transplant patient received the equivalent amount of tissue derived from only two embryonic ventral mesencephali per putamen. In the two post mortem autopsy cases, survival of only modest numbers of dopaminergic neurons were reported (7,000-38,000 per putamen). This stands in contrast to the substantially higher numbers $(82,000-138,000$ per putamen, and up to 208,000 in total in one patient) ${ }^{3}$, alongside a generally more pronounced clinical improvement ${ }^{3.5}$, achieved using conventional neurosurgical techniques and fresh tissue from embryos that had been cool-stored for at most a few days ${ }^{3}$. More generally, the extent of symptomatic improvement over several years after transplantation is closely related to the degree of dopaminergic-cell survival and reinnervation as assessed by positron emission tomography ${ }^{4}$, which is in turn dependent on the amount of tissue implanted as well as the methods of preparation ${ }^{5}$. From this perspective, the numbers reported by Freed et al. are at the lower rather than upper limits of survival for any significant functional benefit to be seen. Most importantly, previous studies ${ }^{5-7}$ have not reported dyskinesia or dystonia in any way comparable in either frequency or magnitude to those reported by Freed et al. Therefore, the lack of more substantial clinical improvement and the occurrence of major side effects are unlikely to be due to the implantation of too much tissue, but rather to a number of major variations of tissue preparation, implantation technique and, possibly, the lack of immunosuppression employed by Freed and colleagues (manuscript submitted and www.nesu.mphy.lu.se/nectar). Unfortunately, too little information is provided to resolve the source of dyskinesias in these patients as the authors have not used standardized assessment protocols for neural transplantation to permit comparability with other studies ${ }^{\text {s. }}$. Moreover, there is no receptor-ligand neuroimaging ${ }^{4}$, which might resolve the relationship between cell survival, reinnervation and receptor sensitivity on which the dyskinetic side effects presumably depend.

In conclusion, the study by Freed et al. provides further evidence that neural transplants can ameliorate parkinsonian symptoms (and that sham surgery has little, or no, lasting effect), but it has opened up more, and not fewer, questions that should foster basic science and clinical research in this exciting fleld of neural repair.

\section{P. Brundin ${ }^{1}$, S. Dunnett ${ }^{3}$, A. BJÖRKLUND $^{2}$ \& G. NIKKHAH ${ }^{4}$ 'Section for Neuronal Survival, "Section for Neurobiology, Wallenberg Neuroscience Center, Lund University, Lund, Sweden \\ ${ }^{3} T$ he Brain Repair Group, School of Biosciences, Cardiff University, Cardiff, Wales, UK ‘Neurosurgical Clinic, Nordstadt Hospital, Hannover, Germany \\ Email: patrik.brundinøneurol.Ju.se}

Freed and colleagues reply-In our doubleblind, placebo-controlled surgical trial of fetal tissue implants for Parkinson disease $^{1}$ (40 patients, 20 transplant subjects and 20 sham surgery controls), we found that transplants survived in $85 \%$ of pa- tients regardless of age and without immunosuppression. In a preplanned division of patients into younger ( $\leq 60$ years) and older (> 60) subjects, younger patients had highly significant improvement in standardized tests of Parkinson disease with the unified Parkinson disease rating scale (UPDRS) total 'off' improving 28\% $(P<0.01)$ and the UPDRS motor 'off' component improving 34\% $(P<0.0005)$. There were no changes in the placebo group. By three years after transplant, the total 'off' scores for the younger group showed further improvement to $38 \%(P=0.004)$. Older patients did not improve as a group, though individuals did. Our study is the largest reported to date and the only controlled trial. Of 20 sham surgery patients, 14 have chosen to receive transplants after the double-blind phase.

The comments of Brundin et al. suggest that the clinical outcome data we have reported are not as good as other reports and that we did not use standard evaluation methods. However, our scoring methods have been standard for up to 30 years $^{810,11}$. To our knowledge, no group has reported clinical results better than we describe.

Brundin et al. contend that the two autopsy cases we reported did not have sufficient cells to produce a clinical effect, but there is no basis for that statement. Our two autopsy patients had respective totals of 44,000 and 63,000 dopaminergic neurons surviving in their transplant tracks. Brundin et al. refer to two postmortem cases in which more embryonic tissue had been transplanted and more cells counted producing "generally more pronounced clinical improvement." That statement is incorrect. Hauser et $a^{\top}{ }^{\tau}$ reported a $32 \%$ improvement in UPDRS total 'off' scores at a mean of 20 months after transplant. Although they presented only six patients in a non-blinded study, their results were nearly identical to those of our controlled trial.

How many cells must survive to get optimum clinical benefit? The answer is unknown. In rat models of Parkinson disease, survival of as few as 200-500 dopaminergic neurons can reverse Parkinson symptoms ${ }^{12}$. Rats normally have 10,000 to 20,000 dopaminergic neurons (humans are estimated to have 500,000 ), so $1-5 \%$ of the normal complement can have a substantial impact on 